\title{
Synthesis, Anti-Inflammatory, and Analgesic Activities of Derivatives of 4-Hydroxy-2-benzoxazolone
}

\author{
Guangjin Zheng, Tieyu Chen, Xing Peng, and Shengjing Long \\ Guangxi Medical University, Guangxi 530021, China \\ Correspondence should be addressed to Xing Peng; xingpeng@gxmu.edu.cn and Shengjing Long; shjlong@126.com
}

Received 6 March 2015; Revised 14 June 2015; Accepted 16 June 2015

Academic Editor: Patricia Valentao

Copyright (C) 2015 Guangjin Zheng et al. This is an open access article distributed under the Creative Commons Attribution License, which permits unrestricted use, distribution, and reproduction in any medium, provided the original work is properly cited.

\begin{abstract}
Benzoxazolones are widely distributed in plants and are of increasing interest for a variety of pharmacological properties, such as detoxification, antibacterial, anti-inflammatory, and analgesic activities and tranquilizers (Michaelidou and Hadjipavlou-Litina, 2005; Doğruer et al., 1998). 4-Hydroxy-2-benzoxazolone (HBOA) is one of the major bioactive compounds in traditional Chinese herb drug Acanthus ilicifolius (Peng and Long, 2006) which has obvious anti-inflammatory and analgesic activities (Huo et al., 2004; Mani Senthil Kumar et al., 2008; Babu et al., 2001). In this research, we used 2-nitroresorcinol as starting material to prepare HBOA with a novel "one-pot-way." Derivatives of HBOA TC-2 TC-4 were obtained via electrophilic reagents and reacted with corresponding primary amines to afford Schiff Base derivatives TC-5 TC-10 for further study. Anti-inflammatory and analgesic activities of those derivatives were determined by using carrageenan-induced rat paw edema test. The analgesic activities of the derivatives were determined by the hot-plate test.
\end{abstract}

\section{Introduction}

In recent years, reports showed Acanthus ilicifolius has obvious anti-inflammatory, analgesic activities, and 4-hydroxy-2 benzoxazolone (HBOA) is one of the major bioactive benzoxazolones compounds [1]. According to modern medicinal theory, $\mathrm{HBOA}$ is a bioisosterism of Chlorzoxazone and has the possibility to be a kind of nonsteroidal anti-inflammatory drugs (NSAIDs). Since benzoxazolones are of bioactivities, many molecular modification and preliminary bioactivity evaluation studies have been performed [1-3]. Several useful compounds have been developed and evaluated for their analgesic properties, anti-inflammatory properties, and antifungal properties [4-7]. Nevertheless, most efforts focused on $\mathrm{N}$-, 4-, 5-, or 6-substituted HBOA. Currently, 7-substituted benzoxazolone derivatives as well as their anti-inflammatory or analgesic properties have been scarcely reported. Therefore, we report the new method for synthesis of HBOA (Scheme 1), some new C-7 HBOA derivatives and their antiinflammatory as well as analgesic activities.

\section{Experimental}

Reagents and solvents used below were obtained from commercial sources. Melting points were measured by X4 micromelting point appearance. IR spectra were obtained with Perkin-Elmer Spectrum 100. The ${ }^{1} \mathrm{H}-\mathrm{NMR}$ and ${ }^{13} \mathrm{C}$ NMR spectra were run on a Bruker AVTCCE AV $600 \mathrm{MHz}$. The mass spectrum was obtained on a Waters AutoSpec Premier P776. Progress of the reaction was monitored by TLC using sheets precoated with UV fluorescent silica gel Merck 60F 254 .

2.1. Preparation of $\mathrm{HBOA}$. To a suspension of $4.0 \mathrm{~g} \mathrm{FeCl}_{3}$. $6 \mathrm{H}_{2} \mathrm{O}$ in $200 \mathrm{~mL}$ methanol, $9.0 \mathrm{~g}$ activated carbon was added and refluxed for 30 minutes. $31.0 \mathrm{~g}$ 2-nitroresorcinol $(0.2 \mathrm{~mol})$ was added and then $36 \mathrm{~mL} 80 \%$ hydrazine hydrate by drops was added in 2 hours. Keep stirring and refluxing for another 4 hours. We filtered all solution to another flask and dried all solvent under vacuum to yield dark syrup. $300 \mathrm{~mL}$ butyl acetate and $60.0 \mathrm{~g}(1 \mathrm{~mol})$ urea were added to the syrup, 


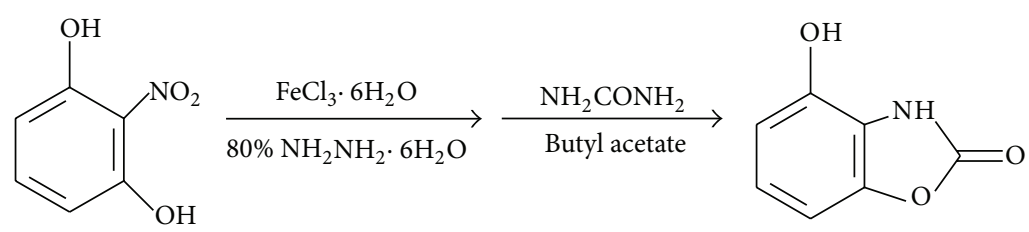

Scheme 1: Methodology for synthesis of HBOA.<smiles></smiles>

SCHeme 2: Methodology for synthesis of TC-2.

stirred at $90^{\circ} \mathrm{C}$ for 5 hours, and then cooled to room temperature. The crude HBOA was obtained by filtration and purified by recrystallization in $80 \%$ ethanol (yield 69.5\%).

2.2. Preparation of TC-2. Compound TC-2 was prepared via chlorination reaction and TC-3 to TC- 4 were prepared via Friedel-Crafts acylation on HBOA. To a solution of $1.51 \mathrm{~g}$ $(0.01 \mathrm{~mol}) \mathrm{HBOA}$ in $15 \mathrm{~mL}$ glacial acetic acid and $2 \mathrm{~mL}$ $(0.024 \mathrm{~mol}) 37 \% \mathrm{HCl}, 1.80 \mathrm{~g}(0.015 \mathrm{~mol}) \mathrm{H}_{2} \mathrm{O}_{2}$ was added in 30 minutes by drops at $45^{\circ} \mathrm{C}$, and then temperature was raised to $80^{\circ} \mathrm{C}$ and stirred for another 2 hours, cooled to room temperature, filtered, and washed with distilled water to obtain compound TC-2 (Scheme 2).

7-Chloro-4-hydroxy-2-benzoxazolone (TC-2). White solid (yield 61\%), m.p. 226 228 C; FT-IR (KBr), v: $3384(\mathrm{OH})$, $3175(\mathrm{NH}), 1748$ (C=O), 1625, 1562, 1492, $1467 \mathrm{~cm}^{-1} ;{ }^{1} \mathrm{H}-\mathrm{NMR}$ (DMSO-d6), $\delta: 11.90(1 \mathrm{H}, \mathrm{s}, \mathrm{N}-\mathrm{H}), 10.38(1 \mathrm{H}, \mathrm{s}, \mathrm{O}-\mathrm{H}), 7.07(1 \mathrm{H}$, $\mathrm{d}, J=8.52 \mathrm{~Hz}, 6-\mathrm{ArH}), 6.85(1 \mathrm{H}, \mathrm{d}, J=8.52 \mathrm{~Hz}, 5-\mathrm{ArH}) .{ }^{13} \mathrm{C}-$ NMR (DMSO-d6), $\delta 154.1$ (2-C), 143.4 (4-C), 138.0 (7a-C), 122.4 (6-C), 116.8 (7-C), 112.2 (3a-C), 103.1 (5-C), EI: $m / z 185$ $\left(\mathrm{M}^{+}, 100 \%\right)$.

2.3. Preparation of TC-3 to TC-4. To slurry of $1.51 \mathrm{~g}(0.01 \mathrm{~mol})$ $\mathrm{HBOA}, 8.0 \mathrm{~g}(0.06 \mathrm{~mol}) \mathrm{AlCl}_{3}$ in $20 \mathrm{~mL} \mathrm{1,2-dichloroethane,}$ and $2 \mathrm{~mL}$ nitrobenzene, $0.04 \mathrm{~mol}$ acyl chloride was added by drops and stirred at rt. for 1 hour. Then temperature was raised to $80^{\circ} \mathrm{C}$ and stirred for another 4 hours. The reaction was quenched by cooling to rt. and pouring the slurry into $300 \mathrm{~mL}$ ice-cold water. Filtered and recrystallized with $90 \%$ ethanol to yield pure compounds TC-3 and TC-4 (Schemes 3 and 4).

7-Acetyl-4-hydroxy-2-benzoxazolone (TC-3). White solid (68\%), m.p. $226 \sim 228^{\circ} \mathrm{C}$; FT-IR (KBr), v: $3358(\mathrm{OH}), 2983$, 2844, 1769 (C=O), 1702 (C=O), 1659, 1625, 1512, 1473, 1427, $1356, \mathrm{~cm}^{-1}$; ${ }^{1} \mathrm{H}-\mathrm{NMR}$ (DMSO-d6), $\delta: 11.88(1 \mathrm{H}, \mathrm{s}, \mathrm{N}-\mathrm{H})$, $11.08(1 \mathrm{H}, \mathrm{s}, \mathrm{O}-\mathrm{H}), 7.46(1 \mathrm{H}, \mathrm{d}, J=8.82 \mathrm{~Hz}, 6-\mathrm{ArH}), 6.74(1 \mathrm{H}$, $\mathrm{d}, J=8.82 \mathrm{~Hz}, 5-\mathrm{ArH}), 2.65\left(3 \mathrm{H}, \mathrm{s}, \mathrm{COCH}_{3}{ }^{*}\right) ;{ }^{13} \mathrm{C}-\mathrm{NMR}$
(DMSO-d6), $\delta: 193.3\left({ }^{*} \mathrm{COCH}_{3}\right), 154.5$ (2-C), 146.9 (4-C), 144.9 (7a-C), 123.7 (6-C), 118.7 (7-C), 113.6 (3a-C), 111.2 (5-C), $30.2\left(\mathrm{CO}^{*} \mathrm{CH}_{3}\right)$. EI: $m / z 193\left(\mathrm{M}^{+}, 78 \%\right)$.

7-Butyryl-4-hydroxy-2-benzoxazolone (TC-4). White solid (72\%), m.p. 168 170; FT-IR (KBr), v: $3138(\mathrm{OH}), 2964,2933$, 2901, 2875, 1777 (C=O), 1748 (C=O), 1659, 1632, 1494, 1472, 1376, 1237, 1156; ${ }^{1} \mathrm{H}-\mathrm{NMR}$ (DMSO-d6), $\delta: 11.87$ (1H, s, N$\mathrm{H}), 10.05(1 \mathrm{H}, \mathrm{s}, \mathrm{O}-\mathrm{H}), 7.48(1 \mathrm{H}, \mathrm{d}, J=8.82 \mathrm{~Hz}, 6-\mathrm{ArH})$, $6.75(1 \mathrm{H}, \mathrm{d}, J=8.82 \mathrm{~Hz}, 5-\mathrm{ArH}), 2.93(2 \mathrm{H}, \mathrm{t}, J=7.14 \mathrm{~Hz}$, $\left.\mathrm{COCH}_{2}{ }^{*} \mathrm{CH}_{2} \mathrm{CH}_{3}\right), 1.64\left(2 \mathrm{H}, \mathrm{m}, \mathrm{COCH}_{2} \mathrm{CH}_{2}{ }^{*} \mathrm{CH}_{3}\right), 0.93$ $\left(3 \mathrm{H}, \mathrm{t}, \mathrm{J}=7.38 \mathrm{~Hz}, \mathrm{COCH}_{2} \mathrm{CH}_{2} \mathrm{CH}_{3}{ }^{*}\right) ;{ }^{13} \mathrm{C}-\mathrm{NMR}$ (DMSOd6), $\delta: 195.7$ ( ${ }^{*} \mathrm{COCH}_{2} \mathrm{CH}_{2} \mathrm{CH}_{3}$ ), 154.5 (2-C), 146.7 (4-C), 144.7 (7a-C), 126.0 (6-C), 123.6 (7-C), 113.4 (3a-C), 111.3 (5C), $43.6\left(\mathrm{CO}^{*} \mathrm{CH}_{2} \mathrm{CH}_{2} \mathrm{CH}_{3}\right), 17.4\left(\mathrm{COCH}_{2}{ }^{*} \mathrm{CH}_{2} \mathrm{CH}_{3}\right), 14.2$ $\left(\mathrm{COCH}_{2} \mathrm{CH}_{2}{ }^{*} \mathrm{CH}_{3}\right)$. EI: $m / z 221\left(\mathrm{M}^{+}, 45 \%\right)$.

2.4. Preparation of TC-5 to TC-10. Compounds TC-5 to TC10 were prepared by reaction with corresponding primary amines to afford Schiff Base derivatives. To a solution of $0.01 \mathrm{~mol}$ starting material in $300 \mathrm{~mL}$ ethanol, $1.5 \mathrm{eq}$ primary amine derivatives were added, refluxed for 3 hours, and then cooled to rt. and filtered and washed with distilled water to yield compounds TC-5 TC-10.

7-(1-(2-Hydroxyethylimino)ethyl)-4-hydroxy-2-benzoxazolone (TC-5). Yellow solid (91\%), m.p. 270 271 ${ }^{\circ}$ C; FT-IR (KBr), $v: 3429(\mathrm{O}-\mathrm{H}), 2963,2873,1757(\mathrm{C}=\mathrm{O}), 1635,1576,1536$, 1444, 1380, 1300, 1286, ${ }^{1} \mathrm{H}-\mathrm{NMR}$ (DMSO-d6), $\delta: 16.80(1 \mathrm{H}, \mathrm{s}$, $\mathrm{N}-\mathrm{H}), 16.20\left(1 \mathrm{H}, \mathrm{s}, \mathrm{C}_{4}-\mathrm{OH}\right), 7.37(1 \mathrm{H}, \mathrm{d}, J=9.0 \mathrm{~Hz}, 6-\mathrm{ArH})$, $6.46(1 \mathrm{H}, \mathrm{d}, J=9.0 \mathrm{~Hz}, 5-\mathrm{ArH}), 3.69(7 \mathrm{H}, \mathrm{m}), 3.43(1 \mathrm{H}, \mathrm{s})$; ${ }^{13} \mathrm{C}-\mathrm{NMR}$ (DMSO-d6), $\delta: 175.6(\mathrm{C}=\mathrm{N}), 161.2(2-\mathrm{C}), 155.0$ (4-C), 147.2 (7a-C), 125.0 (6-C), 121.2 (7-C), 113.0 (3a-C), 97.3 (5-C), $60.0\left(\mathrm{CN}^{*} \mathrm{CH}_{2} \mathrm{CH}_{2} \mathrm{OH}\right), 48.2\left(\mathrm{CNCH}_{2}{ }^{*} \mathrm{CH}_{2} \mathrm{OH}\right), 15.2$ $\left(\mathrm{CH}_{3}\right)$. EI: $m / z 236\left(\mathrm{M}^{+}, 100 \%\right)$.

7-(1-(2-Hydroxyethylimino)butyl)-4-hydroxy-2-benzoxazolone (TC-6). Light yellow solid (45\%), m.p. 255 257 C; FT-IR (KBr), v: 3431 (O-H), 3323 (N-H), 3027 (=C-H), 2957, 


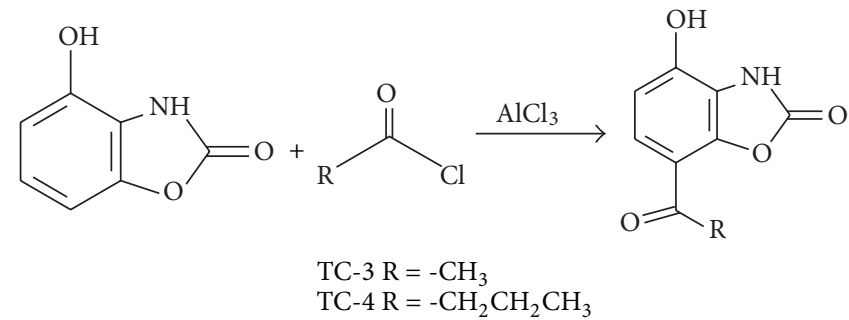

SCHEme 3: Methodology for synthesis of TC-3 and TC-4.<smiles>[R2]CN=C([R])c1ccc(O)c2[nH]c(=O)oc12</smiles>

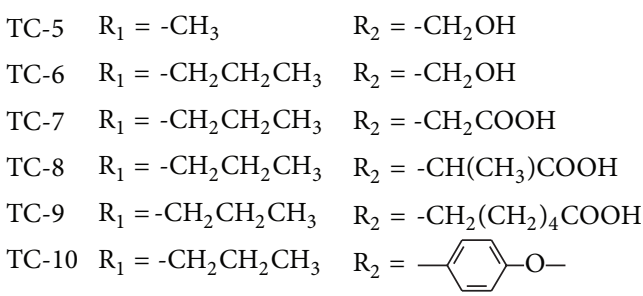

Scheme 4: Methodology for synthesis of TC-3 and TC-4.

2863, 1751 (C=O), 1634, 1617, 1571, 1441, 1383, 1286, 1072, ${ }^{1} \mathrm{H}-\mathrm{NMR}$ (DMSO-d6), $\delta: 16.84(1 \mathrm{H}, \mathrm{s}, \mathrm{N}-\mathrm{H}), 16.30(1 \mathrm{H}, \mathrm{s}$, $\left.\mathrm{C}_{4}-\mathrm{OH}\right), 7.36(1 \mathrm{H}, \mathrm{d}, J=9.0 \mathrm{~Hz}, 6-\mathrm{ArH}), 6.47(1 \mathrm{H}, \mathrm{d}, J=$ $9.0 \mathrm{~Hz}, 5-\mathrm{ArH}), 3.70(4 \mathrm{H}, \mathrm{m}), 3.51(1 \mathrm{H}, \mathrm{s}), 2.88(2 \mathrm{H}, \mathrm{t}, J=$ $2.28 \mathrm{~Hz}), 1.61(2 \mathrm{H}, \mathrm{m}), 1.04(3 \mathrm{H}, \mathrm{d}, J=2.28 \mathrm{~Hz}) ;{ }^{13} \mathrm{C}-\mathrm{NMR}$ (600 MHz, DMSO), $\delta$ : $178.2\left({ }^{*} \mathrm{C}=\mathrm{N}\right), 161.6$ (2-C), 155.0 (4-C), 147.1 (7a-C), 124.8 (6-C), 121.4 (7-C), 111.8 (3a-C), 97.5 (5-C), $60.1\left(\mathrm{CN}^{*} \mathrm{CH}_{2} \mathrm{CH}_{2} \mathrm{OH}\right), 47.9\left(\mathrm{CNCH}_{2}{ }^{*} \mathrm{CH}_{2} \mathrm{OH}\right), 29.4$ ( $\left.\mathrm{CH}_{2} \mathrm{CH}_{2} \mathrm{CH}_{3}\right), 22.0\left(\mathrm{CH}_{2}{ }^{*} \mathrm{CH}_{2} \mathrm{CH}_{3}\right), 14.4\left(\mathrm{CH}_{2} \mathrm{CH}_{2}{ }^{*} \mathrm{CH}_{3}\right)$. EI: $m / z 264\left(\mathrm{M}^{+}, 100 \%\right)$.

2-(1-(4-Hydroxy-2-benzoxazolone-7-yl)butylide-neamino)acetic Acid (TC-7). White solid (56\%), m.p. 80 182 ${ }^{\circ}$ C; FT-IR $(\mathrm{KBr}), v: 3333(\mathrm{O}-\mathrm{H}), 3138(\mathrm{~N}-\mathrm{H}), 2969,2880,1740(\mathrm{C}=\mathrm{O})$, $1721(\mathrm{C}=\mathrm{O}), 1625,1593,1509,1425,1370,1228,1057 ;{ }^{1} \mathrm{H}-\mathrm{NMR}$ (DMSO-d6), $\delta: 13.45$ (1H, s, COO-H), 11.68 (1H, s, N-H), 8.24 $(1 \mathrm{H}, \mathrm{s}, \mathrm{O}-\mathrm{H}), 7.64(1 \mathrm{H}, \mathrm{d}, J=9.0 \mathrm{~Hz}, 6-\mathrm{ArH}), 6.45(1 \mathrm{H}, \mathrm{d}, J=$ $9.0 \mathrm{~Hz}, 5-\mathrm{ArH}), 3.85\left(2 \mathrm{H}, \mathrm{s}, \mathrm{CNCH}_{2}{ }^{*} \mathrm{COOH}\right), 2.95(2 \mathrm{H}, \mathrm{t}, J$ $\left.=7.38 \mathrm{~Hz}, \mathrm{CH}_{2}{ }^{*} \mathrm{CH}_{2} \mathrm{CH}_{3}\right), 1.66\left(2 \mathrm{H}, \mathrm{m}, \mathrm{CH}_{2} \mathrm{CH}_{2}{ }^{*} \mathrm{CH}_{3}\right), 0.95$ $\left(3 \mathrm{H}, \mathrm{t}, J=7.38 \mathrm{~Hz}, \mathrm{CH}_{2} \mathrm{CH}_{2} \mathrm{CH}_{3}{ }^{*}\right) ;{ }^{13} \mathrm{C}-\mathrm{NMR}$ (DMSO-d6) $\delta$ : $177.6\left(^{*} \mathrm{COOH}\right), 172.1\left({ }^{*} \mathrm{C}=\mathrm{N}\right), 158.0(2-\mathrm{C}), 156.6(4-\mathrm{C})$, 147.1 (7a-C), 128.0 (6-C), 115.1 (7-C), 112.7 (3a-C), 110.1 (5-C), $60.1\left(\mathrm{CN}^{*} \mathrm{CH}_{2} \mathrm{COOH}\right), 42.2\left({ }^{*} \mathrm{CH}_{2} \mathrm{CH}_{2} \mathrm{CH}_{3}\right), 18.3$ $\left(\mathrm{CH}_{2}{ }^{*} \mathrm{CH}_{2} \mathrm{CH}_{3}\right), 14.1\left(\mathrm{CH}_{2} \mathrm{CH}_{2}{ }^{*} \mathrm{CH}_{3}\right)$. EI: $m / z 278\left(\mathrm{M}^{+}\right.$, $12 \%)$.
2-(1-(4-Hydroxy-2-benzoxazolone-7-yl)butylide-neamino)propanoic Acid (TC-8). White solid (56\%), m.p. $144 \sim 146^{\circ} \mathrm{C}$; FT-IR (KBr), v: 3352 (O-H), 2966, 2937, 2876, 1721 (C=O), $1702(\mathrm{C}=\mathrm{O}), 1642,1605,1512,1456,1383,1232,1067,802$; ${ }^{1} \mathrm{H}-\mathrm{NMR}$ (DMSO-d6), $\delta: 13.49$ (1H, s, COO-H), 13.16 $(1 \mathrm{H}, \mathrm{s}, \mathrm{N}-\mathrm{H}), 8.14(1 \mathrm{H}, \mathrm{s}, \mathrm{O}-\mathrm{H}), 7.63(1 \mathrm{H}, \mathrm{d}, J=9.0 \mathrm{~Hz}$, 6-ArH), $6.45(1 \mathrm{H}, \mathrm{d}, J=9.0 \mathrm{~Hz}, 5-\mathrm{ArH}), 4.19(1 \mathrm{H}, \mathrm{q}, J=$ $\left.7.2 \mathrm{~Hz}, \mathrm{CNCH}^{*}\left(\mathrm{CH}_{3}\right) \mathrm{COOH}\right), 2.96(2 \mathrm{H}, \mathrm{t}, J=3.66 \mathrm{~Hz}$, $\left.\mathrm{CH}_{2}{ }^{*} \mathrm{CH}_{2} \mathrm{CH}_{3}\right), 1.65\left(2 \mathrm{H}, \mathrm{m}, \mathrm{CH}_{2} \mathrm{CH}_{2}{ }^{*} \mathrm{CH}_{3}\right), 1.33(3 \mathrm{H}$, $\left.\mathrm{d}, J=2.88 \mathrm{~Hz}, \mathrm{CNCH}\left(\mathrm{CH}_{3}{ }^{*}\right) \mathrm{COOH}\right), 0.95(3 \mathrm{H}, \mathrm{t}, J=$ $\left.3.60 \mathrm{~Hz}, \mathrm{CH}_{2} \mathrm{CH}_{2} \mathrm{CH}_{3}{ }^{*}\right)$; ${ }^{13} \mathrm{C}-\mathrm{NMR}(600 \mathrm{MHz}, \mathrm{DMSO})$, $\delta: 195.6\left(^{*} \mathrm{COOH}\right), 176.7\left(^{*} \mathrm{C}=\mathrm{N}\right), 154.5(2-\mathrm{C}), 146.7$ (4-C), 144.7 (7a-C), 126.0 (6-C), 123.5 (7-C), 113.4 (3a-C), 105.5 (5-C), $60.6\left(\mathrm{CN}^{*} \mathrm{CH}\left(\mathrm{CH}_{3}\right) \mathrm{COOH}\right), 43.5\left({ }^{*} \mathrm{CH}_{2} \mathrm{CH}_{2} \mathrm{CH}_{3}\right)$, $30.0 \quad\left(\mathrm{CH}_{2}{ }^{*} \mathrm{CH}_{2} \mathrm{CH}_{3}\right), \quad 17.5 \quad\left(\mathrm{CNCH}\left({ }^{*} \mathrm{CH}_{3}\right) \mathrm{COOH}\right), \quad 14.2$ $\left(\mathrm{CH}_{2} \mathrm{CH}_{2}{ }^{*} \mathrm{CH}_{3}\right) \cdot \mathrm{m} / z 292\left(\mathrm{M}^{+}, 40 \%\right)$.

6-(1-(4-Hydroxy-2-benzoxazolone-7-yl)butylide-neamino) hexanoic Acid (TC-9). Light yellow solid (44\%), m.p. 138.5 140 ${ }^{\circ}$; FT-IR (KBr), v: 3368 (O-H), 2962, 2943, 2874, $1732(\mathrm{C}=\mathrm{O}), 1707$ (C=O), 1637, 1606, 1517, 1465, 1375, 1088, 798; ${ }^{1} \mathrm{H}-\mathrm{NMR}$ (DMSO-d6), $\delta: 13.51$ (1H, s, COO-H), 12.06 $(1 \mathrm{H}, \mathrm{s}, \mathrm{N}-\mathrm{H}), 7.96(1 \mathrm{H}, \mathrm{s}, \mathrm{O}-\mathrm{H}), 7.61(1 \mathrm{H}, \mathrm{d}, J=9.0 \mathrm{~Hz}$, 6-ArH $), 6.43(1 \mathrm{H}, \mathrm{d}, J=9.0 \mathrm{~Hz}, 5-\mathrm{ArH}), 3.11(2 \mathrm{H}, \mathrm{t}, J=$ $\left.6.84 \mathrm{~Hz}, \mathrm{CNCH}_{2}{ }^{*} \mathrm{CH}_{2} \mathrm{CH}_{2} \mathrm{CH}_{2} \mathrm{CH}_{2} \mathrm{COOH}\right), 2.96(2 \mathrm{H}, \mathrm{m}$, 
$\left.\mathrm{CNCH}_{2} \mathrm{CH}_{2} \mathrm{CH}_{2} \mathrm{CH}_{2} \mathrm{CH}_{2}{ }^{*} \mathrm{COOH}\right), 2.21(2 \mathrm{H}, \mathrm{t}, J=7.38 \mathrm{~Hz}$, $\left.\mathrm{CH}_{2}{ }^{*} \mathrm{CH}_{2} \mathrm{CH}_{3}\right), 1.64\left(2 \mathrm{H}, \mathrm{m}, \mathrm{CNCH}_{2} \mathrm{CH}_{2}{ }^{*} \mathrm{CH}_{2} \mathrm{CH}_{2} \mathrm{CH}_{2} \mathrm{COOH}\right)$, $1.52\left(2 \mathrm{H}, \quad \mathrm{m}, \quad \mathrm{CNCH}_{2} \mathrm{CH}_{2} \mathrm{CH}_{2} \mathrm{CH}_{2}{ }^{*} \mathrm{CH}_{2} \mathrm{COOH}\right), \quad 1.45$ $\left(2 \mathrm{H}, \mathrm{m}, \mathrm{CNCH}_{2} \mathrm{CH}_{2} \mathrm{CH}_{2}{ }^{*} \mathrm{CH}_{2} \mathrm{CH}_{2} \mathrm{COOH}\right), 1.31(2 \mathrm{H}, \mathrm{m}$, $\left.\mathrm{CH}_{2} \mathrm{CH}_{2}{ }^{*} \mathrm{CH}_{3}\right), 0.93\left(3 \mathrm{H}, \mathrm{t}, J=7.38 \mathrm{~Hz}, \mathrm{CH}_{2} \mathrm{CH}_{2} \mathrm{CH}_{3}{ }^{*}\right) ;{ }^{13} \mathrm{C}-$ NMR (DMSO-d6), $\delta: 174.9\left({ }^{*} \mathrm{COOH}\right), 172.3\left({ }^{*} \mathrm{C}=\mathrm{N}\right), 158.1$ (2-C), 156.0 (4-C), 155.7 (7a-C), 127.5 (6-C), 115.5 (7-C), 112.5 (3a-C), 110.4 (5-C), $59.4\left(\mathrm{CN}^{*} \mathrm{CH}_{2} \mathrm{CH}_{2} \mathrm{CH}_{2} \mathrm{CH}_{2} \mathrm{CH}_{2} \mathrm{COOH}\right)$, $47.7 \quad\left(\mathrm{CNCH}_{2} \mathrm{CH}_{2} \mathrm{CH}_{2} \mathrm{CH}_{2}{ }^{*} \mathrm{CH}_{2} \mathrm{COOH}\right), \quad 34.1$ ( $\left.{ }^{*} \mathrm{CH}_{2} \mathrm{CH}_{2} \mathrm{CH}_{3}\right)$, $29.6\left(\mathrm{CNCH}_{2}{ }^{*} \mathrm{CH}_{2} \mathrm{CH}_{2} \mathrm{CH}_{2} \mathrm{CH}_{2} \mathrm{COOH}\right)$, $26.4 \quad\left(\mathrm{CNCH}_{2} \mathrm{CH}_{2} \mathrm{CH}_{2}{ }^{*} \mathrm{CH}_{2} \mathrm{CH}_{2} \mathrm{COOH}\right), \quad 24.7$ $\left(\mathrm{CNCH}_{2} \mathrm{CH}_{2}{ }^{*} \mathrm{CH}_{2} \mathrm{CH}_{2} \mathrm{CH}_{2} \mathrm{COOH}\right), 18.3\left(\mathrm{CH}_{2}{ }^{*} \mathrm{CH}_{2} \mathrm{CH}_{3}\right)$, $14.1\left(\mathrm{CH}_{2} \mathrm{CH}_{2}{ }^{*} \mathrm{CH}_{3}\right) \cdot \mathrm{m} / z 334\left(\mathrm{M}^{+}, 32 \%\right)$.

7-(1-(4-Methoxyphenylimino)butyl)-4-hydroxy-2-benzoxazolone (TC-10). Light yellow solid (52\%), m.p. $206 \sim 207^{\circ} \mathrm{C}$; FT-IR (KBr), v: $3438(\mathrm{O}-\mathrm{H}), 2972,2876,2836,1778(\mathrm{C}=\mathrm{O})$, 1631, 1608, 1570, 1510, 1442, 1376, 1071, $1034\left(v_{\mathrm{s}}\left(\mathrm{C}_{7 \mathrm{a}}-\mathrm{O}-\mathrm{C}_{2}\right)\right.$, 850; ${ }^{1} \mathrm{H}-\mathrm{NMR}$ (DMSO-d6), $\delta: 12.09(1 \mathrm{H}, \mathrm{s}, \mathrm{N}-\mathrm{H}), 11.85(1 \mathrm{H}$, s, O-H), $7.54(1 \mathrm{H}, \mathrm{d}, J=8.76 \mathrm{~Hz}, 6-\mathrm{ArH}), 7.08(2 \mathrm{H}, \mathrm{d}, J=$ $\left.8.94 \mathrm{~Hz}, 3^{\prime}, 5^{\prime}-\mathrm{ArH}\right), 6.82(1 \mathrm{H}, \mathrm{d}, J=8.76 \mathrm{~Hz}, 5-\mathrm{ArH}), 6.51$ $\left(2 \mathrm{H}, \mathrm{d}, J=8.94 \mathrm{~Hz}, 2^{\prime}, 6^{\prime}-\mathrm{ArH}\right), 3.79\left(3 \mathrm{H}, \mathrm{s}, \mathrm{OCH}_{3}{ }^{*}\right), 2.74(2 \mathrm{H}$, t, $\left.J=5.76 \mathrm{~Hz}, \mathrm{CH}_{2}{ }^{*} \mathrm{CH}_{2} \mathrm{CH}_{3}\right), 1.58\left(2 \mathrm{H}, \mathrm{m}, \mathrm{CH}_{2} \mathrm{CH}_{2}{ }^{*} \mathrm{CH}_{3}\right)$, $0.83\left(3 \mathrm{H}, \mathrm{t}, J=7.38 \mathrm{~Hz}, \mathrm{CH}_{2} \mathrm{CH}_{2} \mathrm{CH}_{3}{ }^{*}\right) ;{ }^{13} \mathrm{C}-\mathrm{NMR}$ (DMSOd6), $\delta$ : $177.4\left({ }^{*} \mathrm{C}=\mathrm{N}\right), 157.6\left(1^{\prime}-\mathrm{C}_{\mathrm{Ar}}\right), 154.9(2-\mathrm{C}), 151.2(4-\mathrm{C})$, 146.9 (7a-C), $137.2\left(4^{\prime}-\mathrm{C}_{\mathrm{Ar}}\right), 124.5(6-\mathrm{C}), 123.6\left(3^{\prime}, 5^{\prime}-\mathrm{C}_{\mathrm{Ar}}\right)$, 119.6 (7-C), $115.0\left(2^{\prime}, 6^{\prime}-\mathrm{C}_{\mathrm{Ar}}\right), 113.4(3 \mathrm{a}-\mathrm{C}), 100.5(5-\mathrm{C}), 55.8$ $\left(\mathrm{O}^{*} \mathrm{CH}_{3}\right), 31.1\left({ }^{*} \mathrm{CH}_{2} \mathrm{CH}_{2} \mathrm{CH}_{3}\right), 22.6\left(\mathrm{CH}_{2}^{*} \mathrm{CH}_{2} \mathrm{CH}_{3}\right), 14.4$ $\left(\mathrm{CH}_{2} \mathrm{CH}_{2}{ }^{*} \mathrm{CH}_{3}\right) m / z: 326\left(\mathrm{M}^{+}, 38 \%\right)$.

\section{Result and Discussion}

\subsection{Biological Testing}

3.1.1. Analgesic Activity. Analgesic activity was examined by using the hot-plate test protocol $[8,9]$. 120 Webster mice of both sexes weighing 18-22 g were used for study. All animals were fed diet in pellets following standard good laboratory practices. Mice were divided into 12 groups. All of the compounds and the reference drug were suspended in $0.5 \%$ carboxymethyl cellulose (CMC) solution. The first group as negative control received 5\% CMC solution and the second group received aspirin $(\mathrm{g})$ as a reference drug, while the other groups received the 10 test compounds. Mice were dropped gently in a dry glass beaker of $1 \mathrm{dm}^{3}$ capacity maintained at $50.5-55^{\circ} \mathrm{C}$. Normal reaction time in seconds for all mice was determined at time intervals of 30,60 , and 90 minutes; this is the interval extending from the instant the mouse reaches the hot beaker till the animal licks its feet or jumps out of the beaker (dose $10 \mathrm{mg} / \mathrm{kg}$ ). The relative potencies to aspirin $(\mathrm{g})$ were then determined (Table 1). Mice were maintained under $12 \mathrm{~h}$ light/dark cycles with controlled temperature $\left(28^{\circ} \mathrm{C}\right)$.

HBOA and TC-3, TC-5, TC-7, TC-8, and TC-9 exhibited more petent analgesic than aspirin. Compounds TC-3, TC-8, and TC-9 showed around twice the activity of aspirin after 90 minutes.
TABLE 1: Analgesic activities of test compounds.

\begin{tabular}{lccc}
\hline \multirow{2}{*}{ Comp. number } & \multicolumn{3}{c}{ Comparative analgesic potency to aspirin after } \\
& $30 \mathrm{~min}$ & $60 \mathrm{~min}$ & $90 \mathrm{~min}$ \\
\hline time in minutes & $1.88 \pm 0.09$ & $1.10 \pm 0.16$ \\
TC-2 & $1.16 \pm 0.03$ & $1.55 \pm 0.12$ & $0.55 \pm 0.08$ \\
TC-3 & $0.70 \pm 0.09$ & $1.88 \pm 0.13$ & $2.14 \pm 0.14$ \\
TC-4 & $0.37 \pm 0.07$ & $0.80 \pm 0.07$ & $0.87 \pm 0.13$ \\
TC-5 & $0.35 \pm 0.03$ & $1.12 \pm 0.08$ & $1.45 \pm 0.15$ \\
TC-6 & $0.91 \pm 0.05$ & $0.45 \pm 0.09$ & $0.56 \pm 0.09$ \\
TC-7 & $1.01 \pm 0.06$ & $1.20 \pm 0.12$ & $1.35 \pm 0.13$ \\
TC-8 & $0.68 \pm 0.07$ & $0.88 \pm 0.11$ & $1.98 \pm 0.14$ \\
TC-9 & $0.60 \pm 0.05$ & $0.91 \pm 0.10$ & $1.81 \pm 0.21$ \\
TC-10 & $0.98 \pm 0.08$ & $0.40 \pm 0.7$ & $0.31 \pm 0.08$ \\
\hline Aspirin & 1.00 & 1.00 & 1.00 \\
\hline
\end{tabular}

All results were significantly different from the standard and normal control. Value at $P=0.05$.

TABLE 2: Anti-inflammatory of test compounds.

\begin{tabular}{lccc}
\hline Comp. number & $\begin{array}{c}\text { Increase in } \\
\text { edema }(\mathrm{mL}) \pm \\
\text { SEM }\end{array}$ & \% protection & $\begin{array}{c}\text { Activity relative } \\
\text { to aspirin }\end{array}$ \\
\hline Control & $1.06 \pm 0.04$ & 0.0 & 0.0 \\
HBOA & $0.82 \pm 0.03$ & 22.5 & 54 \\
TC-2 & $0.64 \pm 0.03$ & 39.6 & 95 \\
TC-3 & $0.48 \pm 0.03$ & 54.7 & 131 \\
TC-4 & $0.73 \pm 0.03$ & 31.1 & 75 \\
TC-5 & $0.57 \pm 0.03$ & 46.2 & 111 \\
TC-6 & $0.85 \pm 0.03$ & 19.8 & 48 \\
TC-7 & $0.73 \pm 0.03$ & 31.1 & 75 \\
TC-8 & $0.46 \pm 0.03$ & 56.6 & 136 \\
TC-9 & $0.78 \pm 0.03$ & 26.4 & 64 \\
TC-10 & $0.95 \pm 0.03$ & 10.4 & 25 \\
Aspirin & $0.62 \pm 0.03$ & 41.5 & 100
\end{tabular}

All results were significantly different from the standard and normal control. Value at $P=0.05$.

3.1.2. Anti-Inflammatory Activity. Anti-inflammatory activity was examined by using carrageenan-induced rat paw edema test [10]. Seventy-two adult Sprague-Dawley rats, weighing $150-180 \mathrm{~g}$, were used. All animals were fed diet in pellets following standard good laboratory practices. The animals were randomly divided into 12 groups of six animals each. All of the compounds and the reference drug were suspended in $0.5 \%$ carboxymethyl cellulose (CMC) solution. The standard drug aspirin and the test compounds were given at a dose of $20 \mathrm{mg} / \mathrm{kg}$. After one hour of the administration of the compounds and standard drug, $0.1 \mathrm{~mL}$ of $1 \%$ carrageenan solution in saline was injected into the subplantar region of the right hind paw of each rat. The right hind paw volume was measured after $3 \mathrm{~h}$ of carrageenan treatment by means of plethysmometer.

The test compounds showed anti-inflammatory activity ranging from $10.4 \%$ to $56.6 \%$, whereas the standard drug aspirin showed $41.5 \%$ inhibition of edema after three hours. Compounds TC-3, TC- 5 , and TC- 8 showed more potent antiinflammatory activity than aspirin (Table 2). 
3.2. Chemistry. In this research, we synthesized compounds TC-2 to TC-10, and their bioactivities were tested by carrageenan-induced rat paw edema and hot-plate test methods. The results show that compounds HBOA and TC3, TC-5, TC-7, TC-8, and TC-9 are active as analgesic and compounds TC-3, TC-5, and TC-8 are active as antiinflammatory agent, while the other compounds are poorly active towards the tested organisms.

\section{Conflict of Interests}

The authors declare that there is no conflict of interests regarding the publication of this paper.

\section{Authors' Contribution}

Tieyu Chen and Guangjin Zheng have equally contributed to this work.

\section{Acknowledgment}

The authors gratefully thank their universities for their equipment and financial support.

\section{References}

[1] B.-L. Deng, M. D. Cullen, Z. Zhou et al., "Synthesis and anti-HIV activity of new alkenyldiarylmethane (ADAM) nonnucleoside reverse transcriptase inhibitors (NNRTIs) incorporating benzoxazolone and benzisoxazole rings," Bioorganic and Medicinal Chemistry, vol. 14, no. 7, pp. 2366-2374, 2006.

[2] Y. Ivanova, G. Momekov, O. Petrov, M. Karaivanova, and V. Kalcheva, "Cytotoxic Mannich bases of 6-(3-aryl-2-propenoyl)2(3H)-benzoxazolones," European Journal of Medicinal Chemistry, vol. 42, no. 11-12, pp. 1382-1387, 2007.

[3] P. Carato, S. Yous, D. Sellier, J. H. Poupaert, N. Lebegue, and P. Berthelot, "Efficient and selective deprotection method for $\mathrm{N}$ protected 2(3H)-benzoxazolones and 2(3H)-benzothiazolones," Tetrahedron, vol. 60, no. 45, pp. 10321-10324, 2004.

[4] K. Shankaran, K. L. Donnelly, S. K. Shah et al., "Inhibition of nitric oxide synthase by benzoxazolones," Bioorganic and Medicinal Chemistry Letters, vol. 7, no. 22, pp. 2887-2892, 1997.

[5] D. D. Erol, M. D. Aytemir, and N. Yuluĝ, "Synthesis and antibacterial and antifungal properties of thiazolinoethyl-2(3H)benzoxazolone derivatives. II," European Journal of Medicinal Chemistry, vol. 31, no. 9, pp. 731-734, 1996.

[6] L. Tang, W.-H. Ma, Y.-L. Ma, S.-R. Ban, X.-E. Feng, and Q.-S. Li, "Synthesis and biological activity of 4-substituted benzoxazolone derivatives as a new class of sEH inhibitors with high anti-inflammatory activity in vivo," Bioorganic \& Medicinal Chemistry Letters, vol. 23, no. 8, pp. 2380-2383, 2013.

[7] L. Tang, S. R. Ban, X. E. Feng, W. H. Lin, and Q. S. Li, "Synthesis and activities of new 4-hydroxy benzoxazolone derivatives," Chinese Chemical Letters, vol. 21, no. 1, pp. 63-66, 2010.

[8] H.-L. Xin, X.-F. Zhai, X. Zheng, L. Zhang, Y.-L. Wang, and Z. Wang, "Anti-inflammatory and analgesic activity of total flavone of Cunninghamia lanceolata," Molecules, vol. 17, no. 8, pp. 8842-8850, 2012.
[9] E. M. Franzotti, C. V. F. Santos, H. M. S. L. Rodrigues, R. H. V. Mourão, M. R. Andrade, and A. R. Antoniolli, "Antiinflammatory, analgesic activity and acute toxicity of Sida cordifolia L. (Malva-branca)," Journal of Ethnopharmacology, vol. 72, no. 1-2, pp. 273-277, 2000.

[10] C. A. Winter, E. A. Risley, and G. W. Nuss, "Carrageenininduced edema in hind paw of the rat as an assay for antiiflammatory drugs," Proceedings of the Society for Experimental Biology and Medicine, vol. 111, pp. 544-547, 1962. 

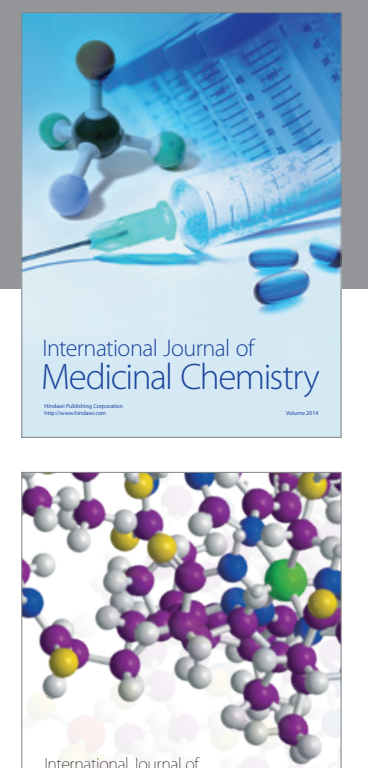

\section{Carbohydrate} Chemistry

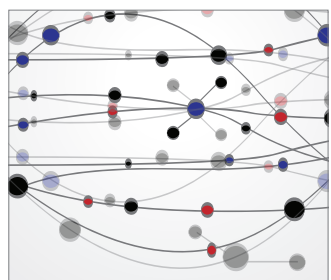

The Scientific World Journal
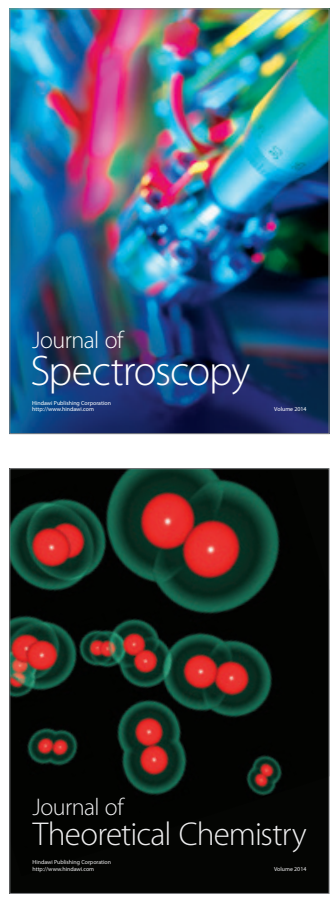
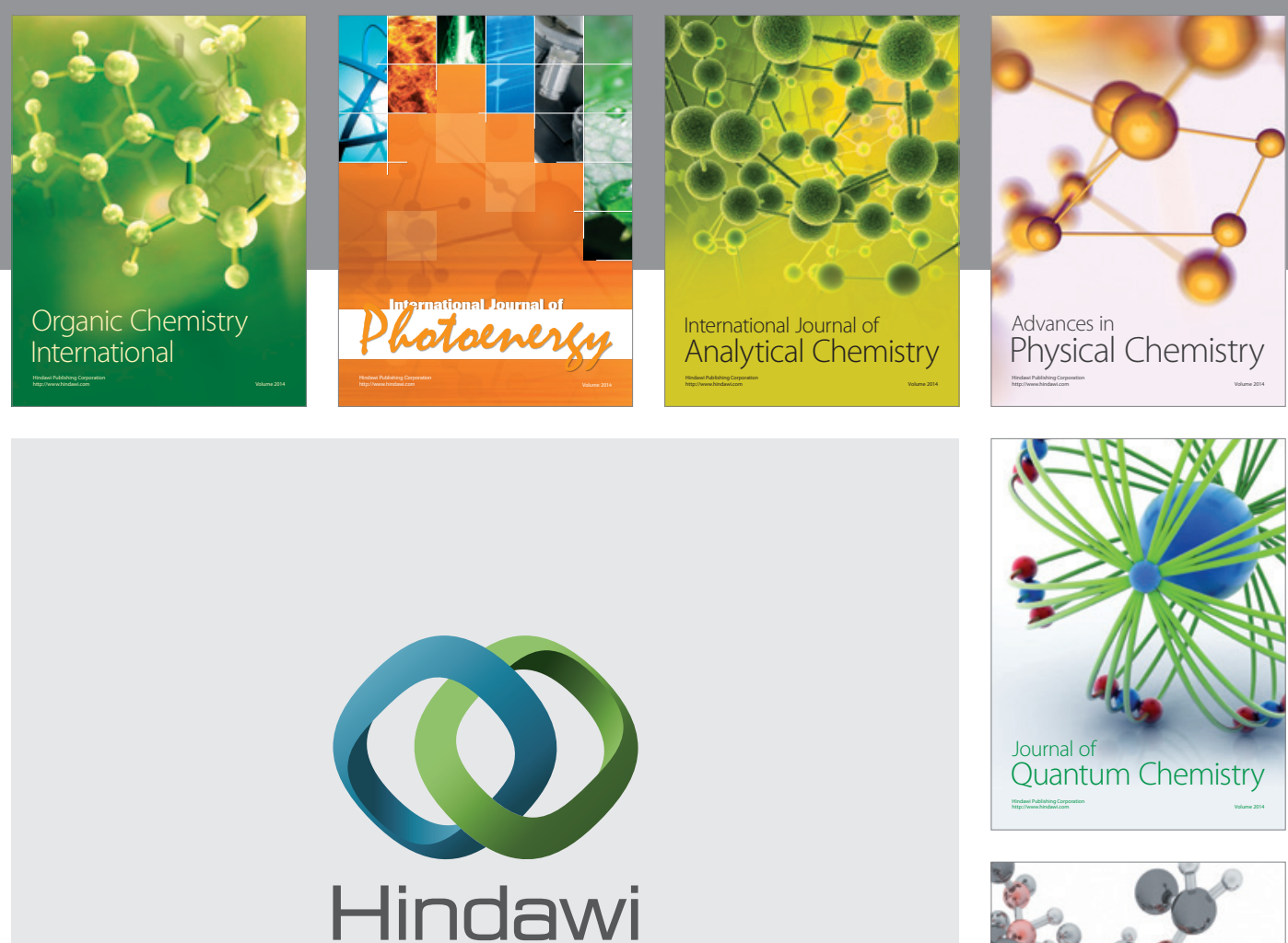

Submit your manuscripts at

http://www.hindawi.com

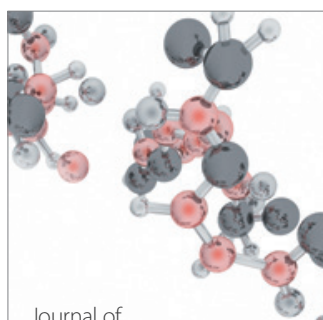

Analytical Methods

in Chemistry

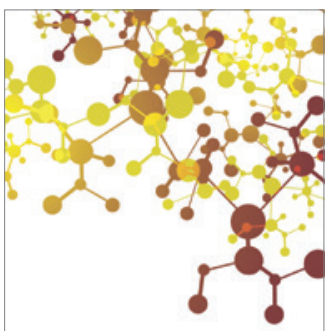

Journal of

Applied Chemistry

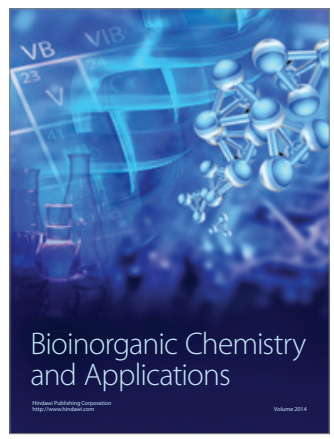

Inorganic Chemistry
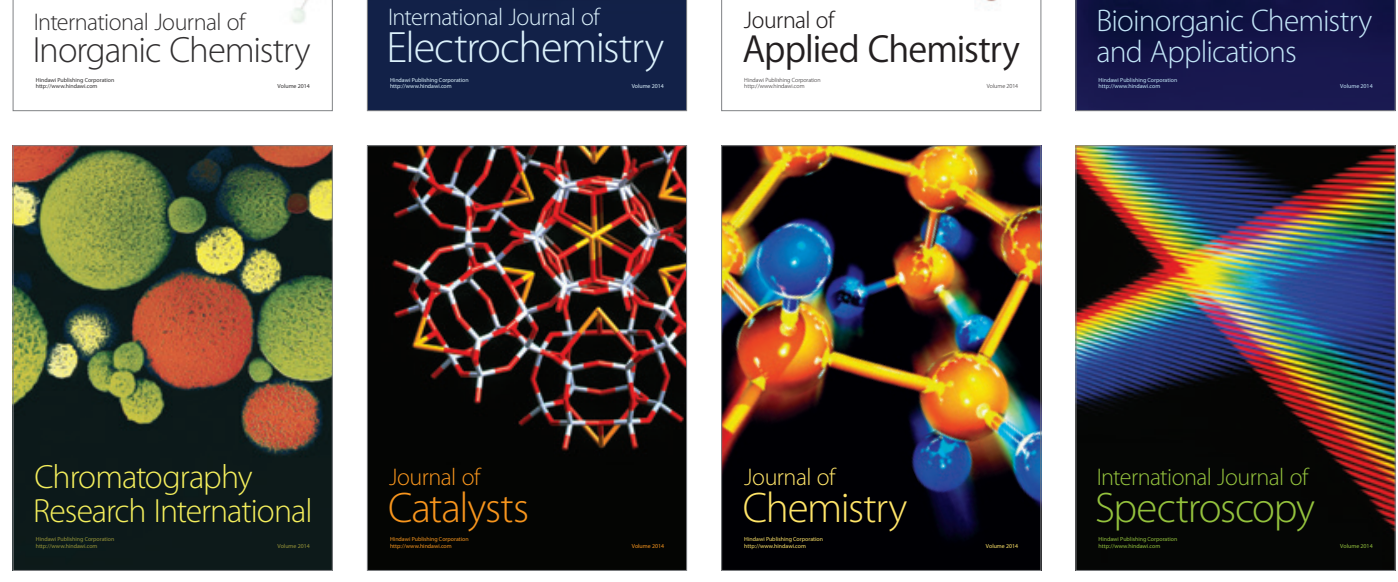